\title{
- Quality of Service in Third-Generation IP-Based Radio Access Networks
}

\author{
Mooi Choo Chuah, Kamesh Medepalli, Se-Yong Park, \\ and Jiansong Wang
}

Currently, the radio access network (RAN) in code division multiple access

(CDMA)-based third-generation (3G) technologies (CDMA2000 and Universal

Mobile Telecommunications Service [UMTS]) is based on asynchronous

transfer mode (ATM). By using the quality-of-service (QoS) guarantees

provided by ATM technology, these $3 G$ systems can efficiently transport

time-sensitive user traffic to different network elements. An effort is

currently under way in several wireless standards bodies to convert this

connection-oriented ATM-based RAN to a connectionless Internet protocol

(IP) network. Such a migration would converge the core and radio access

networks and hence yield cost savings and ease of maintenance. It is also apparent that such a migration imposes new challenges to be solved on the QoS front since IP is, predominantly, a best-effort transport mechanism. In this paper, we explore the QoS support provided by 3rd Generation Partnership Project 2 (3GPP2) standards and discuss several possibilities for using IP transport without degrading the QoS experienced by different traffic types. We provide a detailed description on the DiffServ approach and how it can be used together with multiprotocol label switching (MPLS) technology to provide the QoS guarantees in an IP-based RAN. Finally, we briefly describe how the QoS framework discussed for CDMA2000* can be applied to UMTS based networks. () 2002 Lucent Technologies Inc.

\section{Introduction}

The radio access network (RAN) in the CDMA2000* system [2, 25] (see Figure 1) consists of the transport network from base station (BS) to base station controller (BSC), BSC to packet control function (PCF), and PCF to packet data serving node (PDSN). The connection between BSC/PCF to PDSN is also referred to as the $R$-P interface. The traffic that flows in the RAN is heterogeneous in nature, and some of the traffic types have stringent delay, loss, and jitter requirements. For example, delay for transporting voice frames must be less than a few milliseconds.
Moreover, the variability in the delay (i.e., jitter) must be small for acceptable voice quality. While the reasons for such stringent requirements are primarily due to the inherent nature of conversational traffic, performance-enhancing schemes like soft-handoff that are employed in code division multiple access (CDMA) systems reinforce the need for timely data delivery. On the forward link (i.e., the direction from BS to mobile) traffic frames from different soft-handoff legs need to reach the base transceiver system (BTS) in time so that the mobile station (MS) can receive the

Bell Labs Technical Journal 7(2), 67-89 (2002) @ 2002 Lucent Technologies Inc. Published by Wiley Periodicals, Inc. Published online in Wiley InterScience (www.interscience.wiley.com). • DOI: 10.1002/bltj.10006 


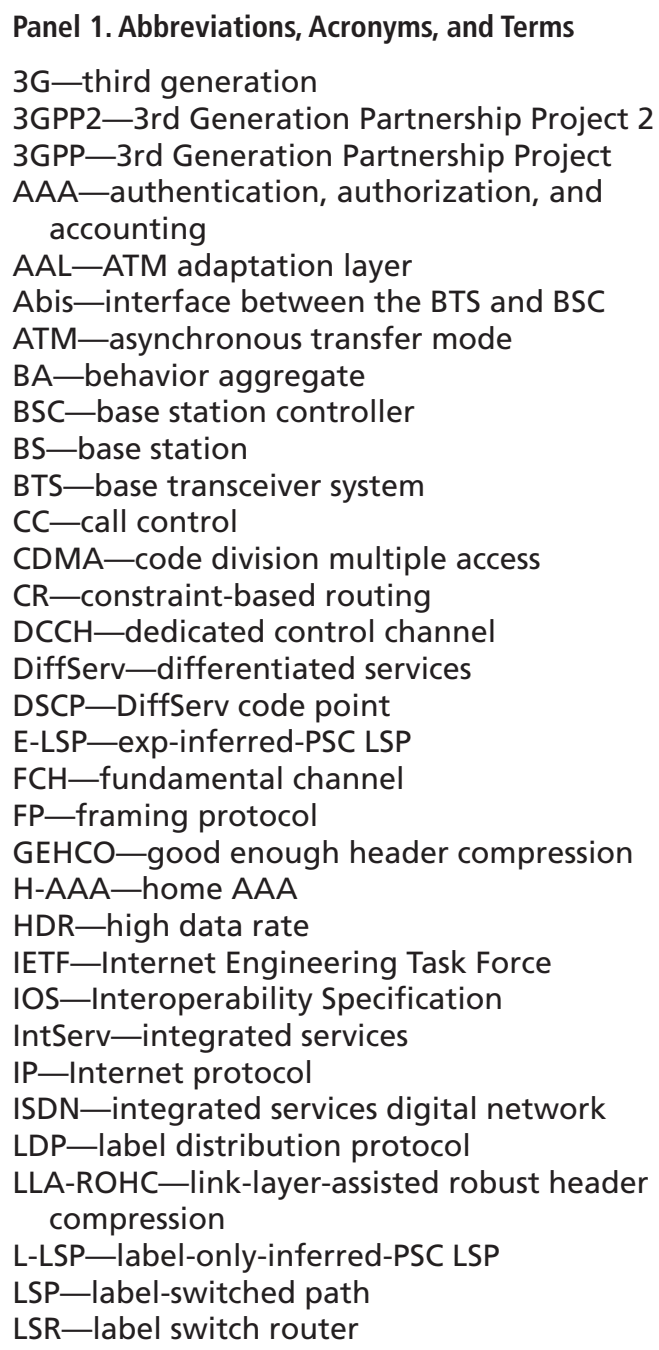

MAC-medium access control MCFTP_multi-channel flow treatment protocol

MPLS-multi-protocol label switching

MS-mobile station

mux-multiplexer

OA\&M-operations, administration, and maintenance

OA-ordered aggregate

PCF-packet control function

PDSN-packet data serving node

PHB - per-hop behavior

PPP_-point-to-point protocol

PSC_-PHB scheduling class

PVC-permanent virtual circuit

QoS-quality of service

RADIUS-remote authentication dial-in user service

RAN-radio access network

RFC-Request for Comments

RLP-radio link protocol

RSVP-resource reservation protocol

RTP-real-time protocol

$\mathrm{SCH}$-supplemental channel

SDU—selection/distribution unit

SO-service option

SR_ID—service reference identifier

TCA - traffic conditioning agreement

$\mathrm{TCP}$ - transmission control protocol

TFT-traffic flow template

UDI-unrestricted digital information

UDP_user datagram protocol

UMTS-Universal Mobile Telecommunications

Service

VolP-voice over IP frames synchronously. Similarly, on the reverse link (i.e., the direction from mobile to BS) traffic frames need to arrive at the frame selector residing in the BSC within predefined delay budgets. In case of data traffic, the delay budgets vary depending on the application type. Interactive traffic like Web browsing can tolerate delay in transfer but not uncorrected transmission errors. An application such as streaming audio or video, however, cannot tolerate delays but can tolerate packet loss arising from transmission errors. In essence, the RAN supports traffic of different delay and loss budgets. Managing the RAN transport network in the presence of these traffic types, in addition to multiple user priority classes and the operations, administration, and maintenance (OA\&M)-type traffic, is quite complex. Current circuit-oriented RAN uses point-to-point connections-that is, asynchronous transfer mode-permanent virtual circuit (ATM-PVC) [2]. Each connection provides guaranteed bandwidth that is engineered to support the expected traffic capacity. Hence delay and jitter can easily be controlled in this environment. 


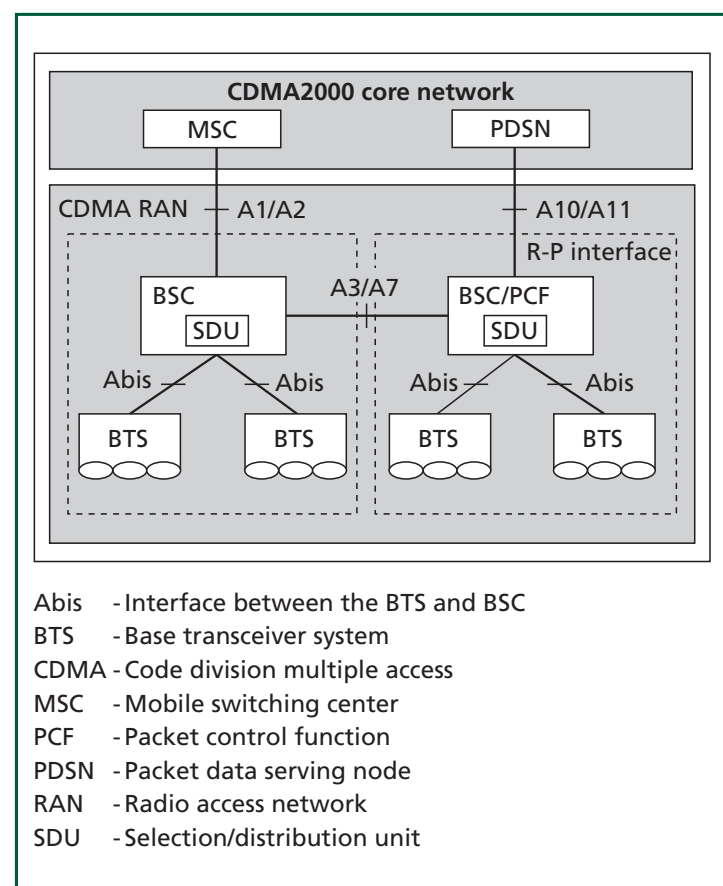

Figure 1.

Network reference models for IOS V4 CDMA2000 RAN.

Several wireless standards bodies are investigating the idea of transforming the current ATM-based RAN of CDMA2000 and UMTS into one that is IP based $[7,13,21]$. One of the primary advantages of employing IP-based transport is the ease of maintenance arising from convergence of core and RAN networks. Other advantages include economic benefits and capacity benefits. IP-based RAN has already been deployed in a $3 \mathrm{G}$ data-only wireless system like the high data rate (HDR) system, also referred to as $1 \mathrm{x}-\mathrm{EV}-\mathrm{DO}$ (CDMA2000 1X evolution in data only mode) [8, 23]. While an IP-based RAN may be a natural choice for a pure data-only system like HDR, it is not an obvious choice when you consider mixed voice and data systems such as CDMA2000 or UMTS. When these $3 \mathrm{G}$ systems migrate from an ATM-based RAN with bandwidth guarantees to one that is based on besteffort IP technology, the task of meeting the QoS requirements for the different applications clearly becomes more challenging.

If an IP RAN relies solely on best-effort IP transport, acceptable quality of service may not be achieved without significantly over-provisioning the network. IP packets are normally subject to jitter arising from variable queuing delay at the routers that process bursty traffic. Moreover, the absence of any packet admission control for best-effort transport can result in congestion at IP routers and packet loss when sufficient buffers are not available for queuing packets.

It is to be noted that supporting traffic classes with varying quality of service (QoS) requirements is not something specific to a wireless environment. A similar problem arises even in the wireline communication networks. To this end, the Internet Engineering Task Force (IETF) has standardized a number of techniques, including integrated services (IntServ) and differentiated services (DiffServ). IntServ (RFC 1633) [11] provides a framework for enabling end-to-end QoS between IP hosts. IntServ uses resource reservation protocol (RSVP) (RFC 2205) [10] to provide a mechanism for reserving transport resources over a signaling plane. DiffServ (RFC 2474, 2475) [9, 19] allows an architecture that can mark packets according to QoS requirements, thus making it possible for IP routers to treat classes of packets differently in terms of priority, traffic shaping, and loss due to congestion.

Currently, multi-protocol label switching (MPLS) is becoming increasingly popular for transport networks that support heterogeneous traffic with guaranteed bandwidth. The primary advantages that MPLS brings are the bandwidth-guaranteeing capability and the traffic-engineering capability. MPLS achieves this by setting up a label-switched path (LSP) that has guaranteed bandwidth using either the RSVP with traffic engineering extensions (RSVP-TE) or constraint-based routing-label distribution protocol (CR-LDP) for signaling.

In general, IP transport QoS in the RAN should be capable of:

- Differentiating user traffic QoS. RAN transport networks must meet the challenge of stringent endto-end quality-of-service (QoS) requirements for multimedia and mission-critical applications via a multiservice IP-based infrastructure. These applications require more predictable end-to-end behavior. 
- Meeting the RAN transport delay, jitter, and loss requirements to maintain user bearer services performance at the radio network layer (e.g., maintenance of frame synchronization, differential delay for soft handoff performance, packet loss rate, and response time of control messages such as power control information flowing between RAN nodes).

Transport QoS in the IP-based RAN is currently under discussion in both 3rd Generation Partnership Project (3GPP) and 3rd Generation Partnership Project 2 (3GPP2) standards bodies, and DiffServ is currently a preferred method for supporting transport QoS (i.e., DiffServ is not made mandatory at the time of this writing). However, DiffServ is a hop-by-hop mechanism at the IP layer and is not designed to guarantee end-to-end QoS. To better achieve transport QoS capabilities comparable to those of ATM, the use of IP over MPLS could be considered. In fact, given the traffic-engineering and bandwidth-guaranteeing abilities of MPLS, it makes itself a readily acceptable mechanism for transporting heterogeneous traffic in the RAN.

At this point, it is worth highlighting that a simpler, alternative approach for providing QoS does exist for the case where backhaul bandwidth is relatively inexpensive. For example, when connecting two network elements, the operator could simply overprovision the transport resources. Moreover, given that air interface is a precious resource in a wireless network, it is preferable to have RAN transport resource availability be such that the utilization of the air interface is maximized. However, in the remainder of this paper, we assume that such over-provisioning is not done due to economic reasons, and explore the current mechanisms described in the 3GPP2 standards for providing QoS over the air and in the RAN. We propose the use of MPLS, in conjunction with or as a substitute for DiffServ, for providing QoS in a future IP-based RAN of the CDMA2000 system.

This paper is organized as follows. In the next two sections, we provide an overview of CDMA2000 system architecture and some of the important protocol stacks for the existing and evolving RAN topologies. In the later sections, we provide background information on DiffServ and MPLS technologies for providing
QoS in the RAN. We then describe the QoS support specified in IS-835 (Wireless IP Network standard) [3] and IS-707-A-2.12 (Data Service Options for CDMA2000) [24] and discuss how different applications and mobiles can use the RAN for their specific needs. We also provide a brief discussion on how the QoS approach discussed for the CDMA2000 system can be applied to the UMTS system. Finally, we provide some concluding remarks on end-to-end QoS in the IP RAN.

\section{Overview of CDMA2000 Architecture}

The existing Interoperability Specification (IOS) for CDMA2000 [2] network reference architecture is shown in Figure 1. The BTS-BSC-MSC path is for circuit voice service while the BTS-BSC/PCF-PDSN path is for packet data services. The Al interface carries signaling information between the call control (CC) and mobility management (MM) functions of the CDMA2000 MSC and the call control component of the CDMA2000 BS (BSC). The A2 interface carries 64/56 kb/s PCM information (voice/data) or $64 \mathrm{~kb} / \mathrm{s}$ unrestricted digital information (UDI, for ISDN). This traffic is carried between the CDMA2000 MSC and one of the channel element components of the CDMA2000 BS (in the case of an analog air interface), or the selection/distribution unit (SDU) function (in the case of a voice call over a digital air interface).

The A3 interface carries coded user information (voice/data) and signaling information between the SDU function and the channel element component of the CDMA2000 BS (BTS). This is a logical description of the endpoints of the A3 interface. The physical endpoints are beyond the scope of this IOS specification. The A3 interface is composed of two parts: signaling and user traffic. The signaling information is carried across a separate logical channel from the user traffic channel, and it controls the allocation and use of channels for transporting user traffic. The A7 interface carries signaling information between a source CDMA2000 BS and a target CDMA2000 BS. The A10 interface carries user traffic between the CDMA2000 RAN and the PDSN. The Al 1 interface carries signaling information between the CDMA2000 RAN and the PDSN. 


\section{Overview of CDMA2000 ATM- and IP-Based Protocol Stacks}

Table I shows the existing protocol stacks for various reference interfaces in the CDMA2000 system. It can be noted that the emphasis is on the ATM connectivity. For example, most signaling messages are sent over transmission control protocol (TCP) over IP over ATM adaptation layer 5 (AAL5) over ATM and bearer traffic over ATM adaptation layer 2 (AAL2) over ATM in the RAN. The exceptions are the Al signaling interface that is carried over narrowband Signaling System 7 (SS7) protocol stack and the A10/A11 interfaces that are carried over IP/Ethernet links.

Table II shows the proposed protocol stacks for the evolved CDMA2000 RAN. Note that layer 3 is IP for all the interfaces, regardless of signaling or bearer traffic. Hence it is an all-IP-based network reference model. All signaling and bearer messages will be carried over IP internetwork. Especially Al signaling interface will use one of the IETF SIGTRAN specifications for carrying SS7 user signaling over the IP transport network. Note that compressed user datagram protocol (UDP) over IP (RFC 2507) [14] and/or compressed real-time protocol (RTP) over UDP/IP (RFC 2508) [12] may be used in the IP RAN to improve the transport efficiency, due to limited transport resources in the RAN. Previous performance studies have shown that IP transport, when used with IP header compression, can yield about 10 to $50 \%$ gain in transport efficiency depending on the traffic type considered.

\section{The Q0S Framework in IP and MPLS Networks}

Before we discuss the QoS approach for the IP-based RAN, we provide some background information on the QoS framework in IP and MPLS networks.

\section{The DiffServ Framework}

The DiffServ architecture (RFC 2475) [9] provides a scalable QoS framework for classifying different flows into differentiated service classes and delivering such differentiated services to the different user flows according to the defined forwarding behaviors. The DiffServ framework is such that traffic flows with higher priority are treated somewhat better than those with lower priority (better in the sense of lower drop

Table I. Protocol stacks of IOS V4.x reference interfaces.

\begin{tabular}{|c|c|c|c|c|c|c|c|c|}
\hline \multicolumn{5}{|c|}{ Signaling Traffic } & \multicolumn{4}{|c|}{ Bearer Traffic } \\
\hline Abis & A1 & A3 & A7 & A11 & Abis & $\mathrm{A} 2$ & A3 & A10 \\
\hline Signaling & Signaling & Signaling & Signaling & Signaling & $\begin{array}{l}\text { User } \\
\text { traffic }\end{array}$ & $\begin{array}{c}56 / 64 \mathrm{~kb} / \mathrm{s} \\
\text { PCM }\end{array}$ & $\begin{array}{l}\text { User } \\
\text { traffic }\end{array}$ & $\begin{array}{l}\text { User } \\
\text { traffic }\end{array}$ \\
\hline TCP & SCCP & $\mathrm{TCP}$ & $\mathrm{TCP}$ & UDP & FP & FP & FP & GRE \\
\hline IP & MTP3 & IP & IP & IP & SSSAR & TDM & SSSAR & IP \\
\hline AAL5 & MTP2 & AAL5 & AAL5 & \multirow[t]{2}{*}{ L2 } & AAL2 & & AAL2 & \multirow[t]{2}{*}{ L2 } \\
\hline ATM & MTP1 & ATM & ATM & & ATM & & ATM & \\
\hline L1 & & L1 & L1 & L1 & L1 & L1 (DS0) & L1 & L1 \\
\hline
\end{tabular}

AAL-ATM adaptation layer

Abis-Interface between the BTS and BSC

ATM-Asynchronous transfer mode

BSC-Base station controller

BTS-Base transceiver station

FP-Framing protocol

GRE-Generic routing encapsulation

IP-Internet protocol

L1-Layer 1

L2-Layer 2
MTP-Message transfer part

PCM-Pulse code modulation

SCCP_Signaling connection control part

SSSAR-Service specific segmentation and reassembly sublayer

TCP-Transmission control protocol

TDM-Time division multiplexing

UDP-User datagram protocol 
Table II. Proposed protocol stacks of IOS V5.x reference interfaces.

\begin{tabular}{|c|c|c|c|c|c|c|c|c|c|}
\hline \multicolumn{6}{|c|}{ Signaling Traffic } & \multicolumn{4}{|c|}{ Bearer Traffic } \\
\hline Abis & \multicolumn{2}{|c|}{ A1 } & A3 & A7 & A11 & Abis & $\mathrm{A} 2$ & A3 & A10 \\
\hline \multirow[t]{3}{*}{ Signaling } & \multicolumn{2}{|c|}{ Signaling } & Signaling & Signaling & Signaling & $\begin{array}{l}\text { User } \\
\text { traffic }\end{array}$ & EVRC & $\begin{array}{l}\text { User } \\
\text { traffic }\end{array}$ & $\begin{array}{l}\text { User } \\
\text { traffic }\end{array}$ \\
\hline & SCCP & SUA & & & & FP & RTP & FP & \\
\hline & M3UA & & & & & & & & \\
\hline $\begin{array}{l}\text { SCTP } \\
\text { (or TCP) }\end{array}$ & \multicolumn{2}{|c|}{ SCTP } & $\begin{array}{c}\text { SCTP } \\
\text { (or TCP) }\end{array}$ & $\begin{array}{c}\text { SCTP } \\
\text { (or TCP) }\end{array}$ & UDP & UDP & UDP & UDP & GRE \\
\hline IP & \multicolumn{2}{|c|}{ IP } & IP & IP & IP & IP & IP & IP & IP \\
\hline L2 & \multicolumn{2}{|c|}{ L2 } & L2 & L2 & L2 & L2 & L2 & L2 & L2 \\
\hline L1 & \multicolumn{2}{|c|}{ L1 } & L1 & L1 & L1 & L1 & L1 & L1 & L1 \\
\hline
\end{tabular}

Abis-Interface between the BTS and BSC

SCCP-Signaling connection control part

EVRC-Enhanced variable rate coder

SCTP-Stream control transmission protocol

FP-Framing protocol

SUA-SS7 SCCP-user adaptation layer

IP-Internet protocol

TCP-Transmission control protocol

L-Layer

RTP-Real-time protocol

M3UA-MTP3 user adaptation layer

UDP-User datagram protocol

probability during periods of congestion and lower latency in processing). Besides performing packet routing and forwarding functions, all IP nodes in the DiffServ domain (a domain typically refers to a network managed by the same administrative organization) may also include two additional functional components: the classifier and the conditioner. The classifier is an entity that selects packets based on the content of packet headers according to defined rules. The conditioner is an entity that performs traffic conditioning functions and it may contain meters, policers, shapers, and markers. The traffic conditioning function is typically deployed at edge nodes of a DiffServ domain. Figure 2 shows the basic functions that can be applied for different components in a DiffServ network. Nodes within the network, such as routers, perform the functions of scheduling and classification.

The DiffServ architecture uses a field in the IP header, called the DiffServ field, as the DiffServ code point (DSCP) for classification of packets from different traffic flows. This field is part of the type of service field in IPv4 and the traffic class octet in IPv6. All the IP packets crossing a link and requiring the same
DiffServ behavior are said to constitute a behavior aggregate (BA). At the ingress node of a DiffServ domain, the packets are classified and marked with a DSCP that corresponds to their BA. The set of BAs

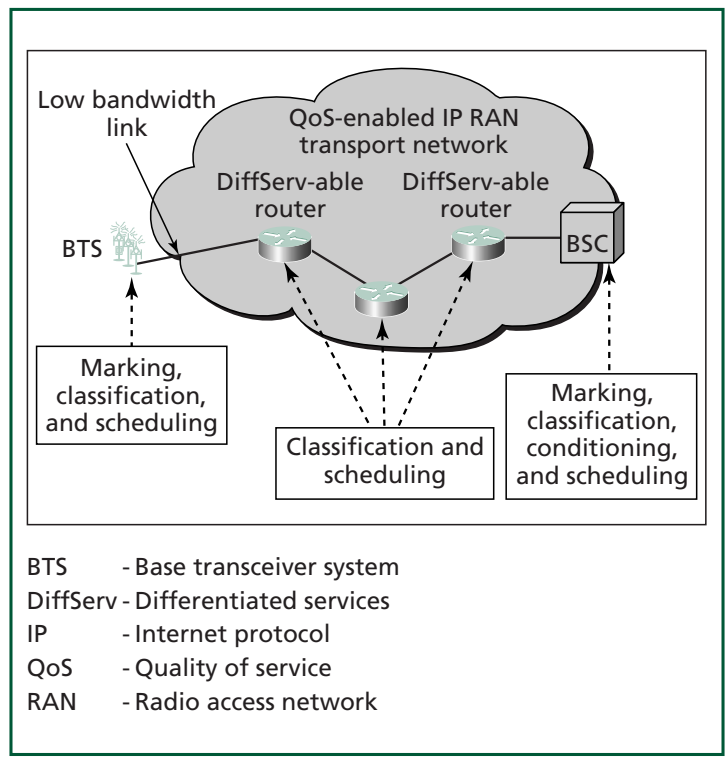

Figure 2.

DiffServ-enabled IP RAN. 
that share an ordering constraint are referred to as an ordered aggregate (OA). A DSCP is an index to a perhop behavior (PHB) in the forwarding treatment table. A PHB is a description of the externally observable forwarding treatment applied at a differentiated service-compliant node. PHBs are specified in terms of resource (e.g., buffer, bandwidth) priority relative to other PHBs, or in terms of their relative observable traffic characteristics (e.g., delay, loss). A PHB descriptor is derived from a set of transport QoS parameters. Transport QoS parameters may include (but are not limited to) the following usage parameters:

- Traffic classes,

- Forwarding priority,

- Expected packet loss rate,

- Expected delay bounds,

- Expected throughput,

- Expected packet mis-insertion rate, and

- $\quad$ Expected peak forwarding rate.

A PHB may also include a specification for traffic conditioning. Traffic conditioning functions include metering, shaping, policing, and marking the packet flows. Traffic conditioning functions are used to conform to the requirements defined in the traffic conditioning agreement (TCA). Thus, a traffic conditioner may alter the temporal characteristics of the traffic behavior aggregate. Currently, the two recognized IETF standard PHB scheduling classes are assured forwarding (RFC 2597) [16] and expedited forwarding (RFC 2598) [17]. A PHB scheduling class (PSC) is a set of one or more PHBs that are applied to the BAs belonging to a given OA. For example, AF1x is a PSC comprising of AF11, AF12 and AF13 PHBs. EF is an example of PSC consisting of a single PHB, the EF PHB. The EF PSC is intended for "premium service," to achieve a low-loss, low-latency, low-jitter, assured bandwidth, and end-to-end service through DiffServ domains. Such a service would appear to endpoints like a point-to-point connection or a "virtual leased line," which is similar to ATM CBR service.

The second standardized PSC, assured forwarding, is a means for the DiffServ domain provider to offer different levels of forwarding assurances for IP packets received from a customer. Traffic may exceed the subscribed profile but the excess traffic is not guaranteed to have the same probability of delivery as the traffic that is within the subscriber's profile. Currently, four AF classes are defined, namely, AFlx, $\mathrm{AF} 2 \mathrm{x}, \mathrm{AF} 3 \mathrm{x}$, and AF4x (where $\mathrm{x}=1,2,3$ ). Each AF class in a given node is allocated different forwarding resources (buffer space and bandwidth). IP packets are assigned to one or more AF classes according to the services to which the customer has subscribed. In each AF PHB class, IP packets are marked with one of three possible drop precedence values. In case of network or node congestion, the drop precedence of a packet determines the relative importance of the packet in the AF class. A congested DiffServ node is more protective of packets with a low drop precedence value, by preferably discarding packets with a higher drop precedence value.

\section{MPLS and the Advantages of QoS Guarantees and Traffic Engineering}

In existing IP networks, as an IP packet travels from one router to the next, each router makes an independent forwarding decision for that packet by analyzing the packet's header and using route entries generated by the network layer routing algorithm it runs. So choosing the next hop router comprises two functions: the first is to partition all packets into sets of forwarding equivalence classes (FECs); the second is to map FEC into a next hop. MPLS (RFC 3031) [22] is a new technology that assigns a particular FEC to a particular packet just once, as the packet enters the network. The FEC to which the packet is assigned is encoded as a label. This label is sent along with the packet to the next hop. At subsequent hops, there is no further analysis of the packet's network layer header. The label is used as an index to a table that specifies the next hop and a new label. The old label is replaced with the new label and the packet is forwarded to the next hop. In order to distribute these labels, IETF defines two signaling protocols: Label distribution protocol (LDP) [4] and RSVP (RFC 2205) [10]. Moreover, the enhancement to LDP that allows explicit routes and traffic engineering capabilities to be specified is referred to as the constraint-based routed LDP (CRLDP) [5]. The enhancement to RSVP to provide such traffic engineering capability is referred to as 
RSVP traffic engineering (RSVP-TE) [6]. We now describe some possible options for setting up LSPs and the corresponding routing protocol preference.

- Hop-by-hop label assignment-In this case, LSP setup requests are routed according to the nexthop routing toward the destination of the data flow. Both LDP and RSVP can be used for this approach.

- Downstream, unsolicited label distribution-The egress label switch router (LSR) distributes the label to be used to reach a particular host. In addition, if the label distribution method is Ordered Control, each upstream LSR distributes a label further upstream and effectively builds a tree rooted at each egress LSR. LDP is more suitable for this mode of label distribution.

- Explicit route specification-If an ingress LSR wants to set up an LSP that does not follow the next-hop routing path, it must use a label distribution protocol that allows specification of an explicit route. CR-LDP and RSVP-TE are two protocols that can be used to set up an explicit route.

- $\quad$ LSP with service guarantees-An ingress LSR may also want to set up an LSP that provides a particular level of service by reserving resources (e.g., bandwidth, QoS) at each intermediate LSR along the path. CR-LDP and RSVP-TE are two protocols that allow downstream on demand label distribution. Such protocols allow the network elements to request for specific service guarantees.

\section{Combining DiffServ and MPLS}

As described in the previous subsection, DiffServ architecture is primarily designed to provide relative QoS. Hence traffic flow with a higher priority is treated somewhat better (for example, in terms of lower drop probability during periods of congestion, smaller latencies in processing) than the one with lower priority. On the other hand, MPLS is designed to provide end-to-end QoS guarantees using prenegotiated LSPs. Moreover, MPLS offers the advantage of traffic engineering naturally. During the LSP set-up phase, RSVP-TE can be used to distribute traffic in the network so that overall performance can be maximized.
Incorporating differentiated services into MPLS is an active research area in the IETF MPLS Working Group. A solution for supporting the DiffServ BA over an MPLS network is provided in [18]. There are two types of LSPs defined in conjunction with DiffServ. These are commonly referred to as exp-inferred-PSC LSPs (E-LSPs) and label-only-inferred-PSC LSPs (L-LSPs) [18]. The L-LSP approach is intended to carry a single OA per LSP while E-LSPs allow multiple OAs to be carried over a single LSP. In case of E-LSPs, the EXP bits of the MPLS shim header are used by the LSR to determine the PHB to be applied to the packet. The mapping from EXP field to PHB for a given LSP is explicitly signaled at label set-up or it relies on preconfigured mapping. In case of L-LSPs, the PSC is explicitly signaled during label establishment so that after label establishment, the LSR can exclusively infer the PSC from the label value (hence EXP bits are not of significance for deriving the PSC). Several enhancements to RSVP-TE and CR-LDP are required to support E-LSPs.

In summary, we have so far discussed DiffServ, MPLS, and the schemes for combining the two. With regard to the IP-based RAN, either one of these three options could be used, although the preferred approach is DiffServ-enabled MPLS. By using a DiffServenabled MPLS RAN, the operators have the advantage of enjoying QoS guarantees (resulting from MPLS) along with differential treatment and standards compliance (DiffServ may be made mandatory in 3G systems). Moreover, operators may first employ DiffServ for QoS differentiation and then employ MPLS for QoS guarantees in a phased manner. On the other hand, it may turn out that for the given traffic levels, traffic types, and the bandwidth provisioned, DiffServ is indeed sufficient. The exact choice depends on several factors including interoperability compliance, traffic levels and types, and economic studies.

\section{Review of QoS Support in the CDMA2000 System}

In this section, we provide a summary of the QoS support as described in the two major CDMA2000 standards. The first standard-the Wireless IP Network Standard (IS-835) [3]-describes the end-to-end point-to-point protocol (PPP) link service and the 
R-P bearer service. In addition to providing the different registration and authentication procedures for simple IP and mobile IP services, it describes the QoS support over the R-P interface ( $\mathrm{R}-\mathrm{P}$ interface is defined as the combination of A10 and All interfaces). The second standard-the Data Service Options in CDMA2000 (IS-707-A-2.12) [24]-describes the air-interface QoS classes and the corresponding QoS parameter negotiation.

\section{QoS at the PPP Level and on the R-P Interface}

In this section, we describe the different QoS classes based on the application type and how multiple applications can share the same PPP connection by using multiple service instances.

QoS classes based on application type. The wireless bearer service classes available in $3 \mathrm{G}$ networks like UMTS/CDMA2000 are conversational, streaming, interactive and background classes [1, 3]. Characteristics of these wireless bearer service classes are listed below:

- Conversational (real-time) class: The types of applications that fall under this traffic class include voice over IP and video conferencing. Fundamental QoS characteristics for this class are:

- A need to preserve the timing relation (i.e., absolute delay and delay variation) between information entities of the stream, and

- Conversational pattern (i.e., highly interactive and extremely low response delay).

- Streaming class: The types of applications that fall under this traffic class include streaming video and audio. Fundamental QoS characteristics for this class are:

- Unidirectional continuous streams, and

- A need to preserve unidirectional timing relation (variation) between information entities of the streams.

- Interactive class: The types of applications that fall under this traffic class include Web browsing, data base retrieval, and server access. Fundamental QoS characteristics for this class are:

- Query and response pattern,

- A need to preserve timing relation at certain levels, and

- A need to reliably deliver the payload content.
- Background class: The types of applications that fall under this traffic class include background download of e-mails. Fundamental QoS characteristics for this class are:

- The destination is not expecting the data within a certain time, and

- A need to reliably deliver the payload content. Before we proceed to briefly describe how multiple service instances can be set up in the same PPP session, we describe a few terms that we will often refer to in the rest of this paper.

- Packet data session: This is a period of time during which the mobile maintains a PPP connection to the PDSN. A mobile can have only one PPP connection with the PDSN.

- Packet call: This is a period of time for which the mobile has radio resources allocated. During this period, a mobile's medium access control (MAC) state is in the active state. When the RLP inactivity timer expires, the mobile will release the radio resources including the RLP, Walsh code, and backhaul resources. However, the PPP connection to the PDSN is maintained. One or more packet calls make up a packet data session.

- Service instance: During a packet call, the mobile can initiate multiple service instances. There are three service types, namely:

- SI-TYPE_1, which identifies a service instance with RLP retransmissions to provide reliable transport for background class, interactive class, and streaming class,

- SI-TYPE_2, which identifies a service instance with no RLP overhead to provide spectrally efficient transport for conversational or streaming codecs (e.g., EVRC, SMV) that are synchronous with the CDMA2000 air framing, and

- SI-TYPE_3, which identifies a service instance with non re-transmitting RLP to provide lowlatency but nonreliable transport for streaming class and conversational class.

Multiple service instances within a PPP session. CDMA2000 service options [25] allow voice and data services to be defined and specified independently within the confines of the physical layer and the 
multiplex sub-layer interface (e.g., service option 33 [SO33] is used for data services). The air interface of CDMA2000 is designed to support multiple instances for the same service option. These instances are referred to as service instances. The CDMA2000 data service option specification [24] supports a maximum of six service instances per MS, each of which may have associated radio link protocol (RLP) and/or QoS parameter setting. The MS and RAN identify specific service instances with an identifier that is referred to as the service reference identifier (SR_ID). There is one $\mathrm{R}-\mathrm{P}$ connection for each service instance. A single R-P session is maintained for all the R-P connections associated with an MS. A single PPP session is associated with the R-P session and there is one PPP session per MS.

A service instance may carry multiple flows. A flow is defined as a series of packets that share a specific instantiation of IETF protocol layers-for example, an RTP flow consists of packets belonging to an RTP/UDP/IP protocol instantiation, all of which share the same source and destination IP addresses and UDP port number. The MS uses packet filters to identify flows within a service instance to the PDSN. The PDSN then uses the mapping information to properly map flows to the R-P connection associated with the service instance. IS-835 groups all flows associated with the same MS IP source address over a single service instance onto one traffic flow template (TFT). The MS signals TFTs to the PDSN via a new PPP control protocol, multi-channel flow treatment protocol (MCFTP). The PDSN and MS identify a specific TFT via the mobile's IP address and SR_ID of the associated service instance. Figure 3 provides an illustration of the relationships between MS IP address, PPP session, R-P session, R-P connection, service instance, SR_ID, and TFT.

If the MS wishes to establish multiple service instances, the MS first establishes a PPP service instance of SI-Type_l before establishing secondary service instances of SI-Type_2 or SI-Type_3. Currently, all the service instances have to be of SO33 (for packet data). However, TSG-P Group of 3GPP2 is in the process of defining two new service options for VoIP. These new service options differ in the way RTP/UDP/IP headers are compressed. Link-layer-assisted robust header compression (LLA-ROHC) is used for transparent header compression in Service Option 61 and another new service option would use good enough header compression (GEHCO) for nontransparent header compression. As mentioned earlier, a new Alo connection is established for each service instance. When a MS initiates secondary service instances, it could send a TFT that contains packet filters associated with each of the service instances. The packet filters are used to map specific forward traffic flows to the corresponding service instance. A maximum of 15 packet filters can be defined for each service instance of SI-Type_l; only one packet filter can be defined for each service instance of SI-Type_2; and a maximum of 15 packet filters can be defined for each service instance of SI-Type_3.

\section{QoS Support over the A10 Interface}

IS-835 [3] proposes using DiffServ over the A10 interface. The usage of DiffServ is according to the subscriber QoS profile. The current version of IS-835 (it is to be noted that, at the time of writing, IS-835 is still undergoing several changes in terms of QoS schemes to be used and the appropriate signalling schemes for conveying QoS parameters) specifies a subscriber QoS profile that:

- Provides a maximum air interface data rate associated with an RP connection that a user may establish,

- Provides a maximum differentiated service level that the mobile may mark reverse traffic, and

- Provides a fixed marking for a reverse tunneled traffic (back to the home agent).

The PDSN can assign DSCP (DiffServ code points) to the A10 connections based on the 3 GPP2 differentiated service class options from the home RADIUS server. The 3GPP2 differentiated service class options specify groupings of differentiated service classes. In essence, the PDSN sends packets received from the IP network for a mobile station onto the R-P session for the mobile station in accordance with differentiated class behavior of the packet and the user profile's 3GPP2 differentiated service class options. The PPP frames that carry the user packets are encapsulated in GRE packets, which in turn are carried over IP. 


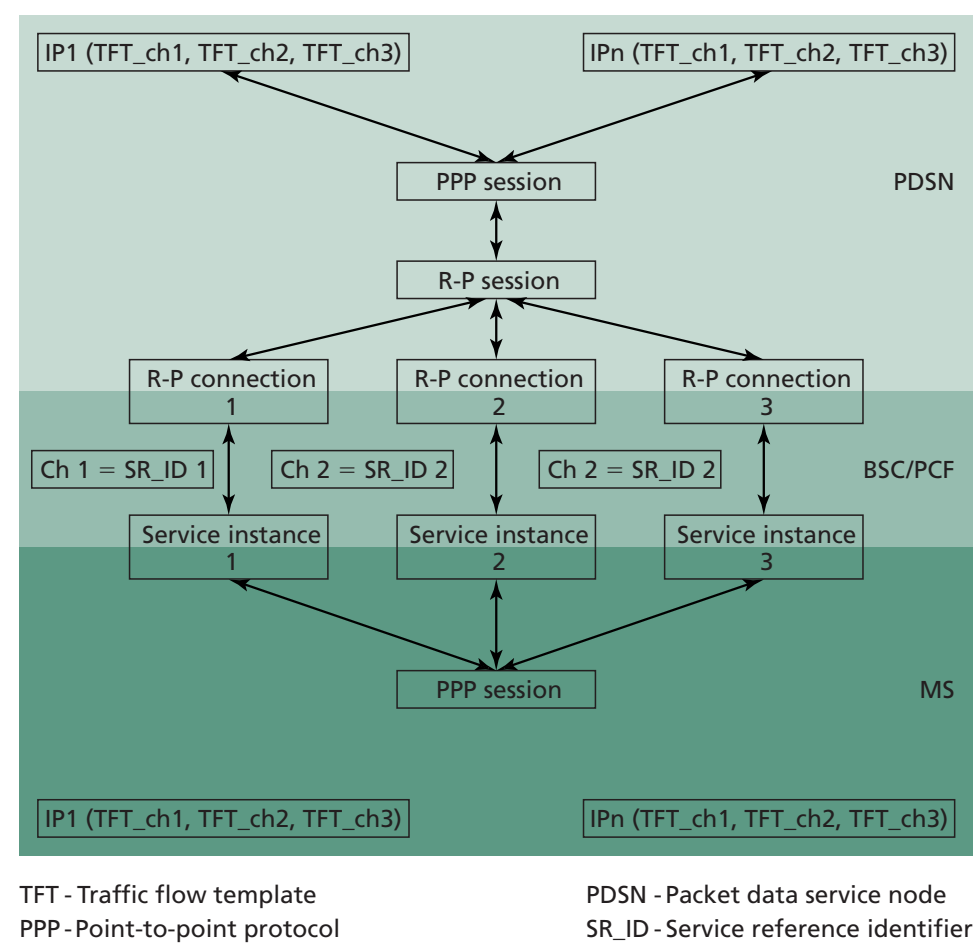

Figure 3.

Relationship between multiple flows, PPP connection, and service instances.

\section{QoS Support over the Air Link}

For the air-link segment of the radio access bearer service, IS-707-A.2.12 [24] allows two QoS modes: assured mode and nonassured mode. A mobile subscribed to the assured mode has the option of sending a set of QoS parameters-for example, the required air-link data rate, acceptable air-link data rate, requested air-link frame error rate, acceptable air-link frame error rate. This set of QoS parameters is referred to as the QOS BLOB. A mobile subscribed to the nonassured mode cannot specify such parameters. However, there exists an option to assign different priority levels (13 to be specific) to the nonassured service. The MAC layer multiplexes traffic from packet data service instances onto physical channels (in fact, the multiplex sub-layer, which is part of the MAC layer of IS-2000, has been defined for this purpose).
The CDMA2000 mobiles can use different traffic channels like fundamental channel (FCH) and supplemental channels (SCHs) in addition to dedicated control channel (DCCH) for carrying traffic. The standard allows a mobile to have two supplemental channels (SCHO and SCH1) in addition to FCH and DCCH. The radio access bearer ( $\mathrm{RAB}$ ) service layer, based on per-call or per-active session requirements from the BSC and the current RF conditions, decides the optimal QoS multiplexing mechanism and the MAC sublayer multiplexing mechanism over the Radio Link. The eventual goal of the RAB service layer is to maximize the RF spectrum utilization, given the constraints of radio link quality and per-flow QoS requirements over the air.

Note that beyond the air-interface parameters, the standard does not define the QoS approach for 
bearer traffic over the backhaul, for example, over the Abis interface. In the next section, we describe an end-to-end QoS approach when the Abis interface is IP based.

\section{End-to-End QoS Approach in IP-Based CDMA2000 RAN}

We start this section by providing a comprehensive view of the hierarchical QoS approach in the CDMA2000 system. Figure 4 shows a logical hierarchy of the end-to-end bearer services in the CDMA2000 system. A similar hierarchical approach for the UMTS system is provided in [15]. The servicelevel agreement (SLA) is negotiated between a mobile subscriber and the wireless service provider. The
SLA typically is expressed in terms of bandwidth, delay, and loss requirements. Such an SLA will be realized using the end-to-end bearer service classes. Some examples of end-to-end bearer service classes are Platinum, Silver, or Bronze services, each with different QoS guarantees. Since an end-to-end connection for a mobile user spans both the wireless and the wireline network, one can further separate the endto-end bearer service classes into wireless bearer service classes and wireline bearer service classes. The wireline bearer service concerns the treatment of user flows (which carry the application contents) over the fixed network that normally has plenty of bandwidth, powerful routers, and highly reliable facilities (e.g., fiber). Therefore, the wireline networks normally

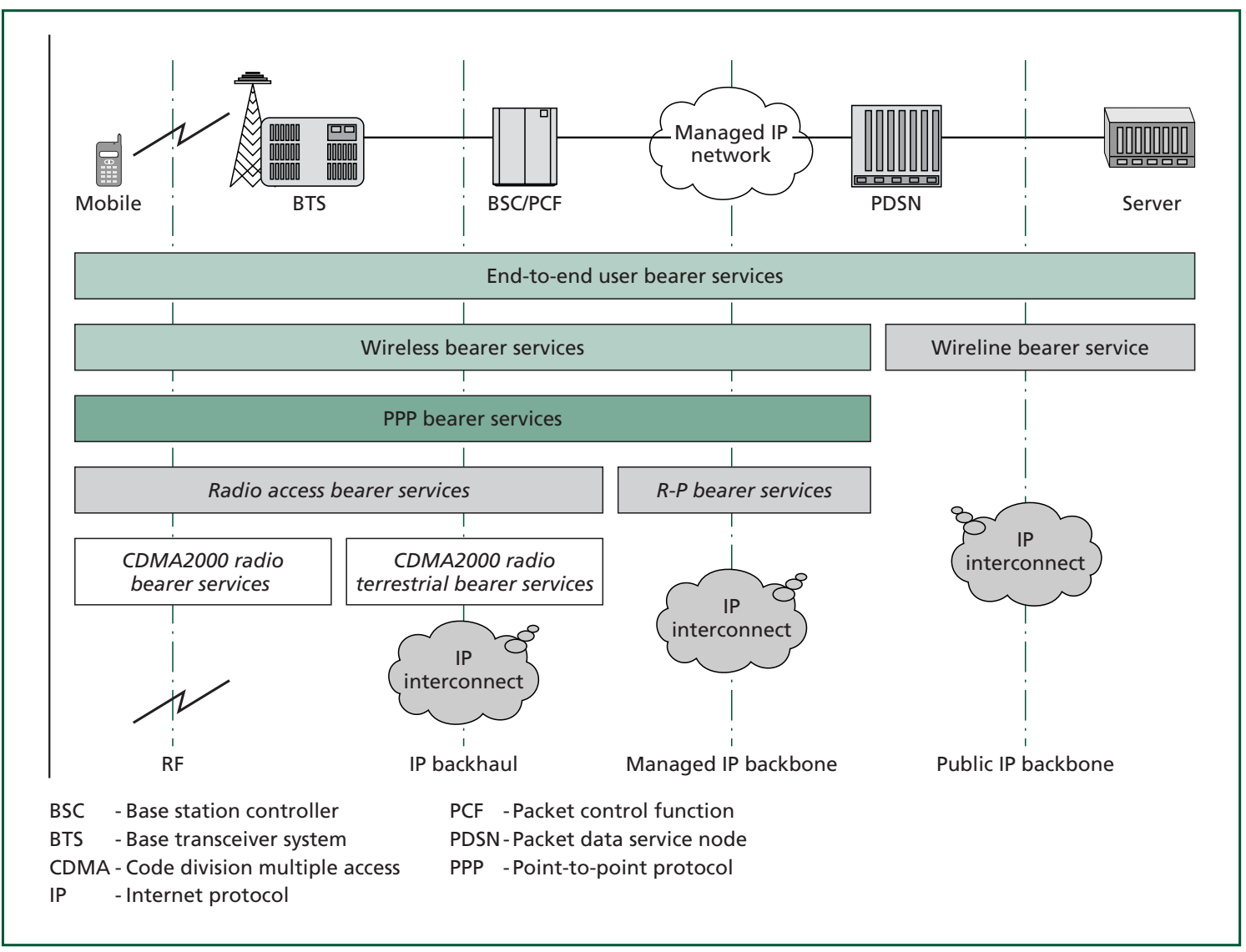

Figure 4.

The layered architecture of the end-to-end user bearer service. 
provide higher QoS performance to its users than that in the wireless networks. If the wireline bearer service is built on top of an IP network, then DiffServ and/or MPLS can be used to provide the required QoS. If the wireline bearer service is built on top of an ATM network, the mature ATM QoS approach using permanent virtual circuits (PVCs) can be used to meet the QoS requirement for the wireline bearer service.

In a CDMA2000 network, the wireless bearer service is built on top of a PPP bearer service. The PPP bearer service consists of the radio access bearer service and the RP bearer service. The radio access bearer service consists of the air-link segment and the segment in the radio access network. The R-P bearer service consists of the segment between the BSC and the PDSN (the Al0 interface in IOS standard).

\section{QoS Approach in the Forward Direction}

There are two approaches for the PDSN to mark the user traffic as well as the GRE/IP packets:

- The PDSN can mark the user traffic and GRE/IP packets based on the QoS BLOB parameter(s) and police or shape the traffic on the Al0 interface in accordance with the differentiated services behavior.

- The PDSN can mark the DSCP of the GRE/IP packet based on the DSCP of the incoming IP packets (see Figure 5).

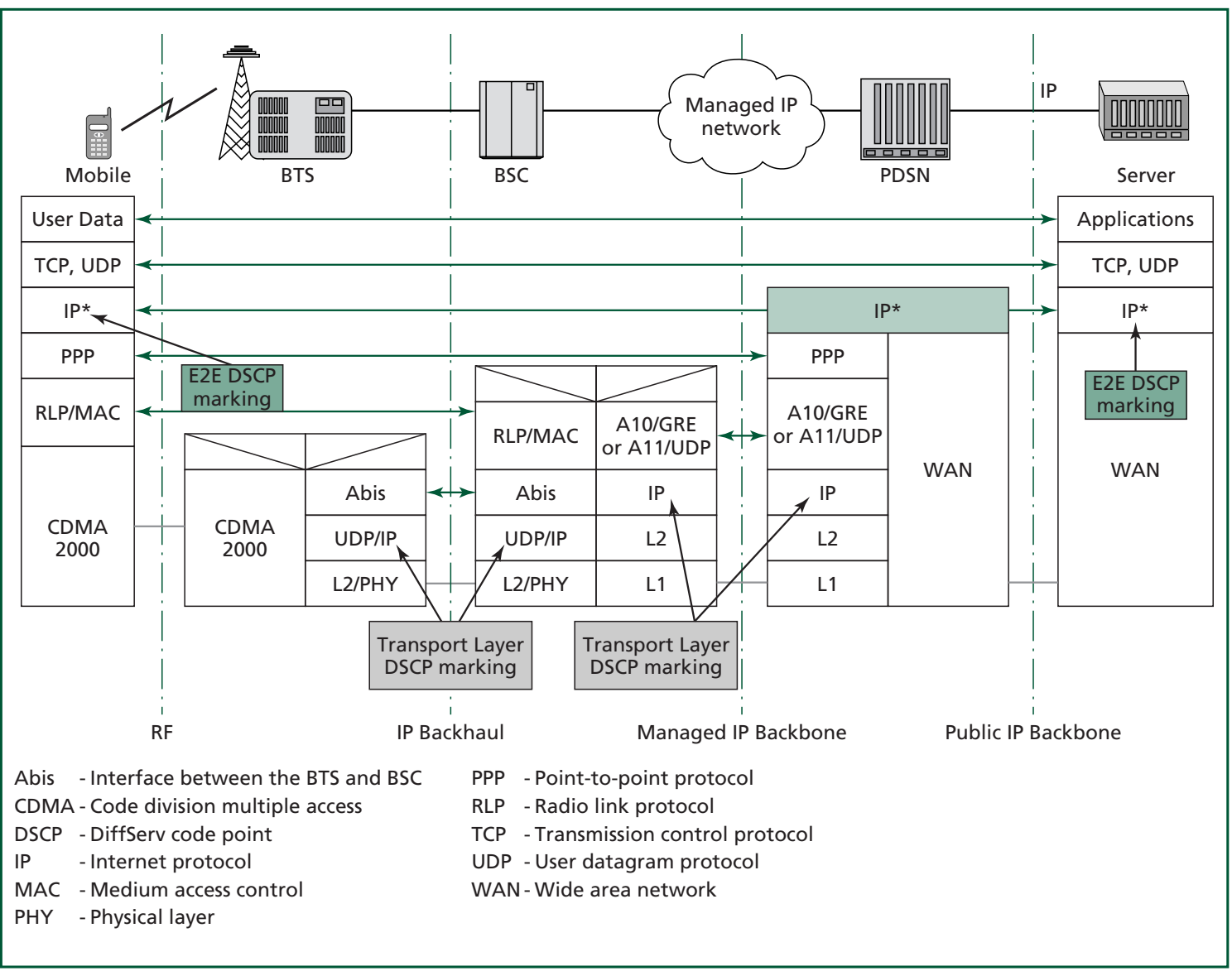

Figure 5.

End-to-end Qos approach on CDMA2000 network. 
The PCF receives the GRE/IP packets from the PDSN and simply maps the outer DSCP on the GRE/IP packet onto a new GRE/IP packet destined to the BSC if the BSC and PCF are not co-located. Note that each service instance is transported on a separate A10 connection. The PCF session ID (PSI) can be used as the GRE key (4 bytes in length). It is possible for the IP packets to be received out of sequence inside the GRE tunnel. For this reason, sequence number option (indicated by S bit in the GRE header) may be turned on so that resequencing is possible. Resequencing also helps the end-to-end performance in case any of the following occur:

- $\quad$ The PDSN is configured to send GRE packets that contain incomplete PPP frames or multiple PPP frames;

- The mobile station negotiates PPP payload compression algorithm that requires PPP frames to be delivered in sequence; or

- The mobile station negotiates Van Jacobson TCP/IP header compression in which the Connection ID may be compressed.

However, if GRE sequence numbering is used at the PDSN, the PDSN will indicate one and only one differentiated service class for all IP packets on the $\mathrm{R}-\mathrm{P}$ session based on the user profile's 3GPP2 differentiated service class options from the home RADIUS server. As for the PDSN, the PCF is not required to police or shape the outbound traffic.

\section{QoS Approach in the Reverse Direction}

When the MS marks IP packets with differentiated services code points (DSCP), the PDSN will ensure that only allowed DSCPs are used as authorized by the home authentication, authorization, and accounting (H-AAA) server in the users 3GPP2 Qos profile. If the H-AAA does not include the 3GPP2 QoS profile, the PDSN will offer a default QoS to the user's packets as provisioned by the service provider. The PDSN will re-mark the packets if the user's markings are not authorized. The BSC/PCF will extract the payload out of an RLP frame, and encapsulate it with GRE/IP header. The BSC/PCF maintains a mapping table to map the GRE tunnel identifier with the service instance identifier. The same mapping table will also include an extra field to indicate what DSCPs will be used to mark the outer IP header of GRE/IP packets. Then, the GRE/IP packets are shipped across the A10 interface to the PDSN. The PDSN will terminate PPP, extract the user's IP packets and re-mark the DSCP code points of the user's IP packets if necessary. Since the delay/ jitter requirements for A10 interface is not as stringent as the Abis interface, the IETF DiffServ approach may be sufficient to provide different QoS treatments to the traffic from different service classes. However, if the BSC/PCF and PDSN are within an IP network where other non-wireless related traffic may be present, then MPLS can be used to provide bandwidth guarantees for the wireless data traffic. The traffic marked with DSCP can be mapped into different MPLS LSPs using the approach described in the previous sections and in [18].

\section{Using DiffServ/MPLS in the IP-Based RAN}

In the existing IOS standard, the CDMA2000 PHY-UP framing protocol (FP) data units are carried using an ATM connection over the Abis/A3 interface while IP/PPP packets are carried using GRE/IP tunnels over the Alo interface. In the future IP-based radio access networks, the FP data units will be carried inside IP packets instead of ATM cells. Since the radio bearer service layer operates in a synchronous fashion but the IP layer operates in an asynchronous fashion, extra care needs to be taken to ensure the voice/data calls meet their QoS requirements. For example, the delay, jitter, and loss requirements across the Abis/ Inter-BSC interfaces are more stringent than the A10/A11 interfaces because the soft handoff scenarios mandate that the FP data units arrive at relevant base stations within certain time windows in order to enjoy the benefits of soft-combining. Thus, a careful design that incorporates a DiffServ-enabled and/or MPLS-enabled network, as described in previous sections, is necessary in order to make an IP-based RAN feasible.

\section{QoS Approach across Abis/A3 Interfaces}

Consider that a packet data user has activated one service instance of SO33 with SI-Type_1, since it is always the primary service instance type required to carry signaling (e.g., PPP signaling) information. The same service instance can be used for reliable delivery 
of Web/e-mail traffic. Consider now that the user has activated another instance of SO33 with SI-Type_2 for streaming applications needs. Separate A10 connections are used to transport PPP frames over different GRE/IP tunnels to the PCF. Appropriate DSCP code points will be used for the different Al0 connections as discussed in earlier section. Once the traffic arrives at the BSC, the RLP frames from different service instances will be multiplexed in the traffic channels. There are two choices for such multiplexing. We will discuss these two choices, recommend one approach, and then discuss how the QoS approach is designed based on this choice.

The two different approaches for multiplexing RLP frames belonging to different service instances in the multiplex sub-layer are as follows:

- RLP frames belonging to different service instances can be multiplexed onto the same SCH service data unit (SDU). Essentially, the traffic streams from different service classes are treated identically over Abis and over the air. By multiplexing data from several service instances, the MAC scheduler located within the BSC can allocate a single high data rate $\mathrm{SCH}$ to the mobile. Note that a mobile can only have a maximum of two SCHs in parallel and it may end up with a single SCH for a large fraction of time due to limited RF resources. Hence the ability to multiplex traffic from several service instances onto the same traffic channel is useful. However, the disadvantage in combining several streams onto the same traffic channel is the loss in the ability to distinguish the individual streams in the RAN transport network and over the air interface. For example, an SCH frame carrying both Web data and streaming audio would have to be treated as streaming audio, which needs to be transported with minimal delay. In doing so, the system has not capitalized on the delay insensitivity feature of non-real-time traffic.

- The traffic from different service instances, but belonging to the same SI-Type, can be multiplexed onto the same physical channel. Hence, different SI-Types will be using different physical channels. Given that an MS has one FCH, one
DCCH, and two SCHs, making a total of three traffic channels, one can assign each one to a service instance type. Note that DCCH, although a dedicated control channel, can carry user traffic. Clearly, FCH (or DCCH) should be used for packet voice traffic as intended by SI-Type_2 because an MS can only have one instance of SI-Type_2 at any time. Since packet voice is usually of low bit rate (for example, EVRC has a peak rate of $8.55 \mathrm{~Kb} / \mathrm{s}$ ), it can easily be transported on an $\mathrm{FCH}$ (or DCCH). Web traffic, e-mail, and other errorintolerant traffic can be multiplexed onto a single high data rate SCH channel that can serve with an RLP configured for retransmission (SI-Type_1) mode. That leaves an additional SCH that can be used to multiplex several streaming applications using a transparent RLP (SI-Type_3). That way, frames (referred to as muxPDUs in CDMA2000) belonging to streaming applications can be transported with small delay but higher residual error rate than SI-Type_1 traffic.

We recommend using the second approach-that is, mapping the three different service instance types to the three different traffic channels available in CDMA2000 system, since such a mapping will enable differential treatment of the traffic flows. Once such a mapping is performed, marking the DSCP header of the IP packet that encapsulates such a muxPDU is straightforward. It is clear that the FCH and DCCH must be given higher priority than the SCH. This is because the FCH and DCCH carry important signaling information regarding the CDMA2000 system, in addition to carrying delay sensitive voice traffic.

After the FCH and DCCH, the SCH carrying SI-Type_3 traffic (say SCHO) should get the next highest priority so that streaming data can be transported with small delay. However, since RLP is not used in the retransmit mode, the error rate for the streaming data will be higher than that for the FCH traffic. The $\mathrm{SCH}$ carrying the non-real-time data (say, SCH1) is given the least priority and hence can be delayed more than the other two. However, it is important to keep the residual error rate for the non-real-time traffic to be the lowest among the three service instance types. 
Apart from the bearer traffic, there are other types of traffic in the RAN-for example, signaling traffic, OAM, and network management traffic. These should be treated with a priority that is lower than that for $\mathrm{SCH}$. In summary, we propose prioritizing the RAN traffic into the following five categories:

1. User data bearer service with very low latency and highly interactive characteristics. The frame protocols that would be in this category are FCH and DCCH. Real-time audits will also require a high level of attention since they are related to system sanity monitoring and overload control in real time. Hence, they are also included in this highest priority class.

2. User data bearers with low latency and general data services. The frame protocols included in this category normally are the CDMA2000 SCH framing protocols. This category of traffic will have a slightly larger packet-loss rate range, compared with the category I traffic, to improve the air-link spectral efficiency.

3. User signaling bearers, including common signaling channels of CDMA2000 systems and all call control signaling traffic in the RAN.

4. Common auditing, $O A \vartheta M$, and other background traffic.

5. Unspecified or unauthorized user traffic.
In Table III, we provide an appropriate DSCP mapping to be used for each traffic category. Note that DSCP code-points are assigned so that intermediary routers can give different treatments to different IP packets. One can use traffic engineering rules to ensure that the different delay and jitter requirements for Abis/A3 interfaces are met. Once the IP packets are marked with the appropriate DSCP, it is easy to map the DSCP to MPLS LSP, as described in previous sections. However, depending upon the volume of traffic in the RAN and the bandwidth available for this traffic, it is quite possible that DiffServ approach is sufficient to meet the various QoS requirements and that bandwidth guaranteed LSPs are unnecessary. It is also quite possible that DiffServ may just be implemented strictly for interoperability compliance among the networks of different operators and is not implemented within the RAN that is solely owned by an operator. Moreover, by using just DiffServ, the QoS processing and management in the IP backhaul can be kept simple.

In the next subsection, we use three simple IP-based RAN topologies as examples illustrating how DSCP code points are used in the RAN with different layer 2 technologies. In all these topologies, the FP data units formatted by the RAB service layer at the

Table III. Generic traffic-classes and the proposed class DSCP at A3/A7/Abis interfaces.

\begin{tabular}{|c|c|c|}
\hline Traffic Class & Examples & Proposed DSCP \\
\hline $\begin{array}{l}\text { Class 1: Very low latency } \\
\text { and/or conversational flows }\end{array}$ & $\begin{array}{l}\mathrm{FCH}, \mathrm{DCCH} \text { and real-time audit } \\
\text { flows }\end{array}$ & $\begin{array}{l}(101) x x x, \text { e.g., (101)110 } \\
\text { for EF services }\end{array}$ \\
\hline $\begin{array}{l}\text { Class 2: Low latency flows and genera } \\
\text { data services }\end{array}$ & $\begin{array}{l}\text { SCHO (streaming) } \\
\text { SCH1 (Web/e-mail etc.) }\end{array}$ & $\begin{array}{l}(100) x x x(A F 4 x) \\
(011) x x x(A F 3 x)\end{array}$ \\
\hline Class 3: Signaling bearer & $\begin{array}{l}\text { Abis and } A 3 / A 7 \text { signaling, } \\
P C H \text { FP, BCH FP, etc. }\end{array}$ & $(010) x x x(A F 2 x)$ \\
\hline $\begin{array}{l}\text { Class 4: General auditing, } \\
\text { OA\&M, background traffic }\end{array}$ & $\begin{array}{l}\text { BSC OA\&M, BTS OA\&M, } \\
\text { Performance measurement }\end{array}$ & $(001) x x x(A F 1 x)$ \\
\hline $\begin{array}{l}\text { Class 5: Unknown/unspecified/ } \\
\text { unauthorized packets }\end{array}$ & $\begin{array}{l}\text { Mis-inserted packets, excessive } \\
\text { packets outside the user } \\
\text { subscription QoS profile }\end{array}$ & $\begin{array}{l}\text { (000)000 (default-best-effort } \\
\text { delivery) }\end{array}$ \\
\hline \multicolumn{3}{|c|}{$\mathrm{FCH}$-Fundamental channel } \\
\hline $\mathrm{BCH}$-Broadcast channel & \multicolumn{2}{|c|}{ FP-Framing protocol } \\
\hline BSC-Base station controller & \multicolumn{2}{|c|}{ OA\&M-Operations, administration, and maintenance } \\
\hline DCCH_Dedicated control channel & \multicolumn{2}{|c|}{ PCH—Paging channel } \\
\hline EF-Expedited forwarding & \multicolumn{2}{|c|}{$\mathrm{SCH}-$ Supplemental channel } \\
\hline
\end{tabular}


BSC are encapsulated with the UDP/IP header. The UDP port number may be used to identify a connection. The resultant IP packet is properly marked with different DSCPs to obtain the proper treatment in the IP back-haul network.

\section{Some Example Topologies and the Corresponding QoS Solution}

Figure 6 shows the first topology where we have PPP links between BSC and BTSs via narrow-band access links (like $\mathrm{Tl} / \mathrm{E} 1$ ) and hence there is no routing involved. Since the overhead of the UDP/IP header is large, UDP/IP header compression method as defined in RFC 2507 [14] will be used to reduce overhead. In addition, PPPMux (RFC 3153) [20] protocol may be used to multiplex small compressed UDP/IP packets into one PPP frame for transport efficiency. PPPMux is terminated at a router close to the IP-based BSC (the router can be considered part of the IP-based BSC). In this topology, one of the ways to provide QoS differentiation is to use different PPP links or to use multi-class extensions to multi-link PPP, if PPP/HDLC is used. One can also carry PPP frames over AAL2/ AAL5/ATM and use ATM QoS framework to meet the delay and jitter requirements. A default mapping between DSCP and ATM QoS is shown in Table IV in case ATM is chosen as the layer 2 technology for the transport network.

Figure 7 depicts the second topology where we have PPP links connecting BTSs to a first hop router that acts as a concentrator. From the first-hop router, the traffic is then routed through a managed IP network to the BSC. In this topology, the PPPMux/PPP is terminated at the first-hop router. One can decide to perform UDP/IP header decompression at the first hop router and use the DSCP information to provide

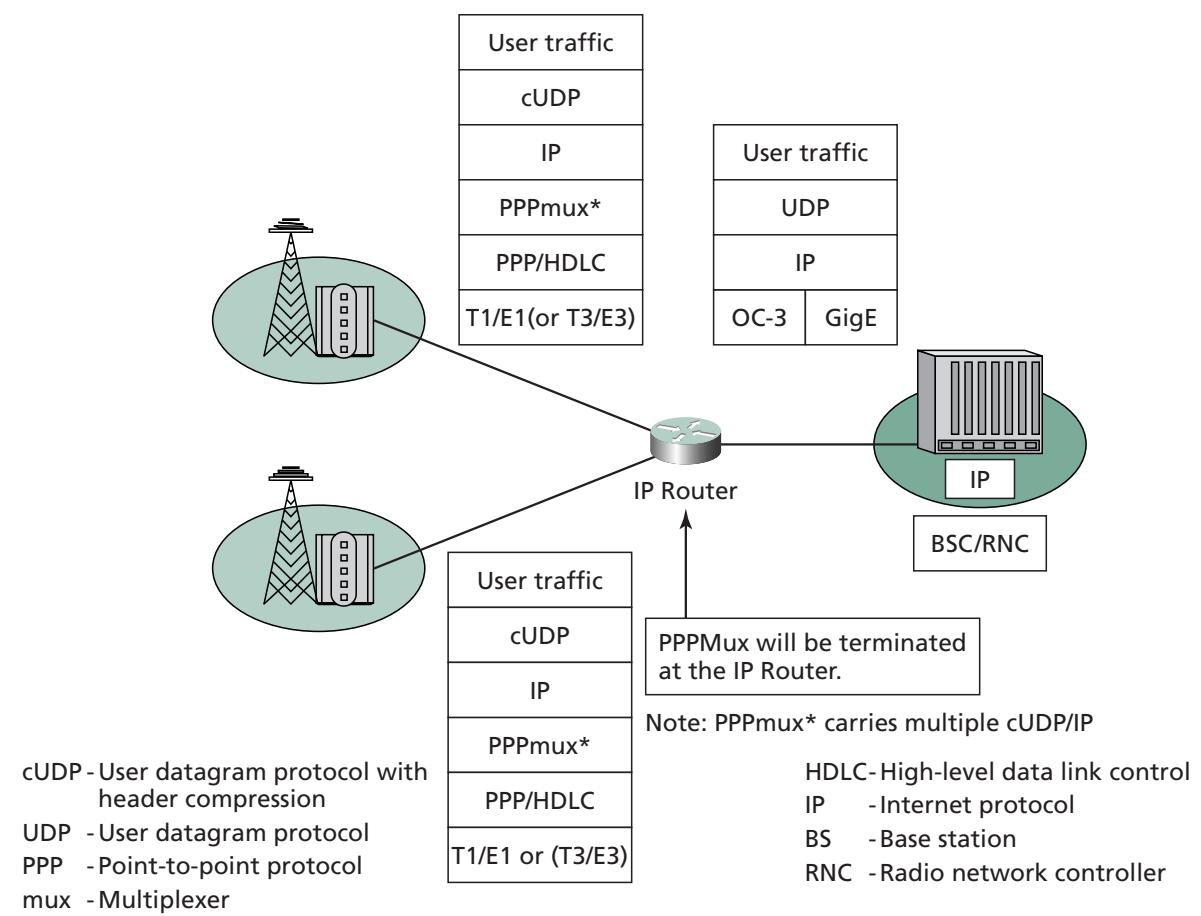

Figure 6.

IP narrowband access using PPPmux terminating at the edge. 
Table IV. A mapping between CDMA2000 IP RAN QoS classes and ATM service classes.

\begin{tabular}{|l|c|c|}
\hline Traffic Class & DSCP & ATM Service \\
\hline Class 1 & EF & CBR/rt-VBR \\
\hline Class 2 & AF4x, AF3x & rt-VBR, nrt-VBR \\
\hline Class 3 & AF2x & nrt-VBR/ABR \\
\hline Class 4 & AF1x & ABR \\
\hline Class 5 & Default/best effort & UBR \\
\hline
\end{tabular}

AF-Assured forwarding

ATM-Asynchronous transfer mode

EF-Expedited forwarding

CBR-Constant bit rate

VBR-Variable bit rate
ABR-Available bit rate

nrt-Non-real time

rt-Real time

DSCP—DiffServ code point

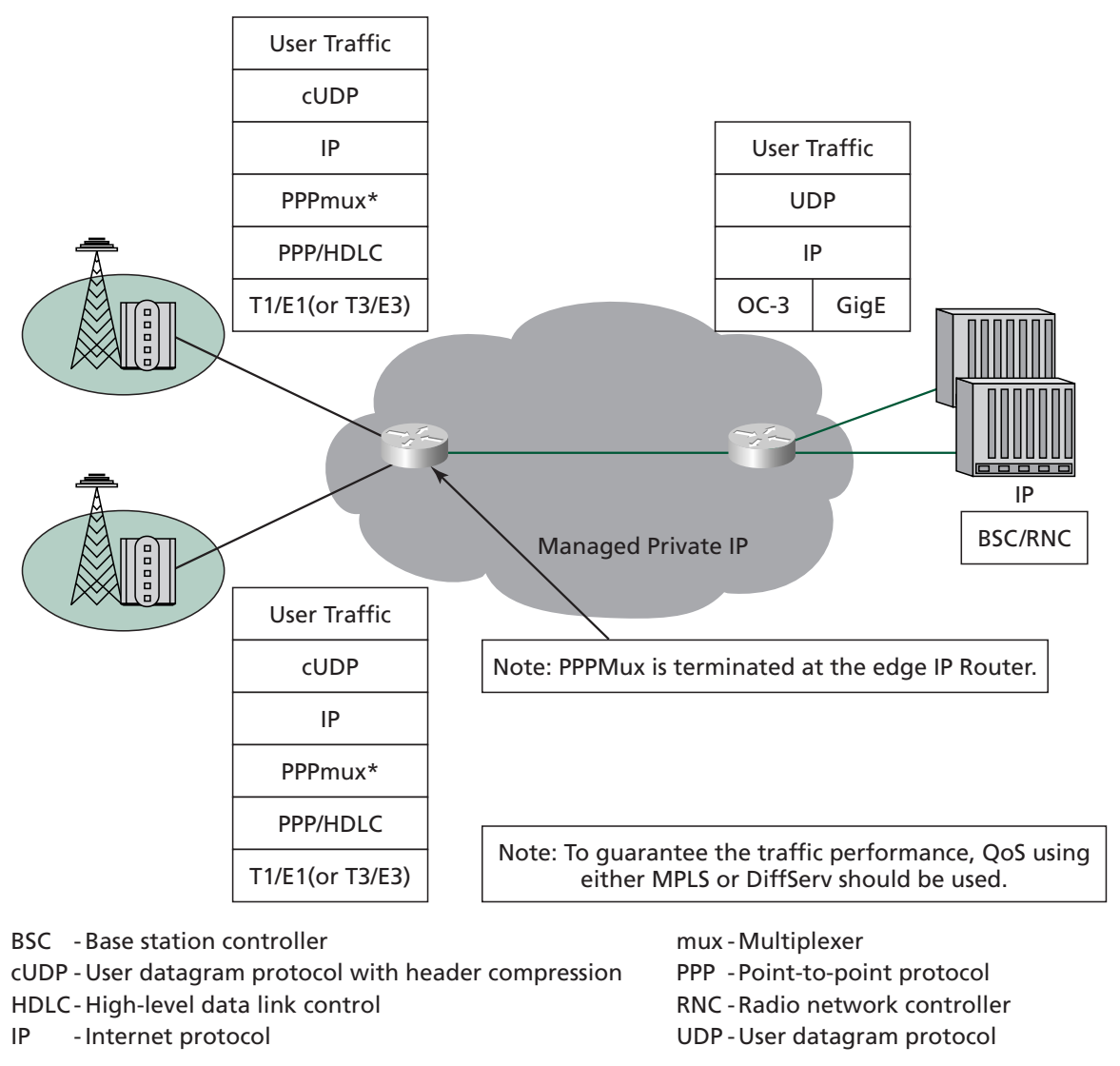

Figure 7.

Narrowband access in last mile using PPPmux.

84 Bell Labs Technical Journal 
different QoS treatments. The penalty in this solution is the high bandwidth cost from the first-hop router to the router next to the IP-based BSC, especially if packet voice traffic is dominant across Abis/A3 interfaces. In addition, the intermediate routers also need to process many small packets (carried in $\mathrm{FCH}$ and $\mathrm{DCCH}$ ). Since DiffServ only provides relative QoS and what we have is stringent delay and loss requirements for Abis/Inter-BSC interfaces, MPLS can be used, as shown in Figure 8. In this case, the first hop router will use the DSCP information to map the traffic into appropriate LSPs as described in the previous sections and in [18].

Figure 9 shows the third topology where gigabit Ethernet links or leased Ethernet services are used to connect the BTSs to the BSC/PCF either directly or via Ethernet switches. One can simply encapsulate FP PDUs using UDP/IP header and deliver such UDP/IP packets over Ethernet. BTS will map DSCP into appropriate $802.1 \mathrm{Q} / \mathrm{p}$ QoS bits so that the packets will be given different QoS treatments. However, we are paying a high overhead cost for small voice payload size. So this solution is only attractive if the bandwidth cost for the Ethernet links is so low that we do not

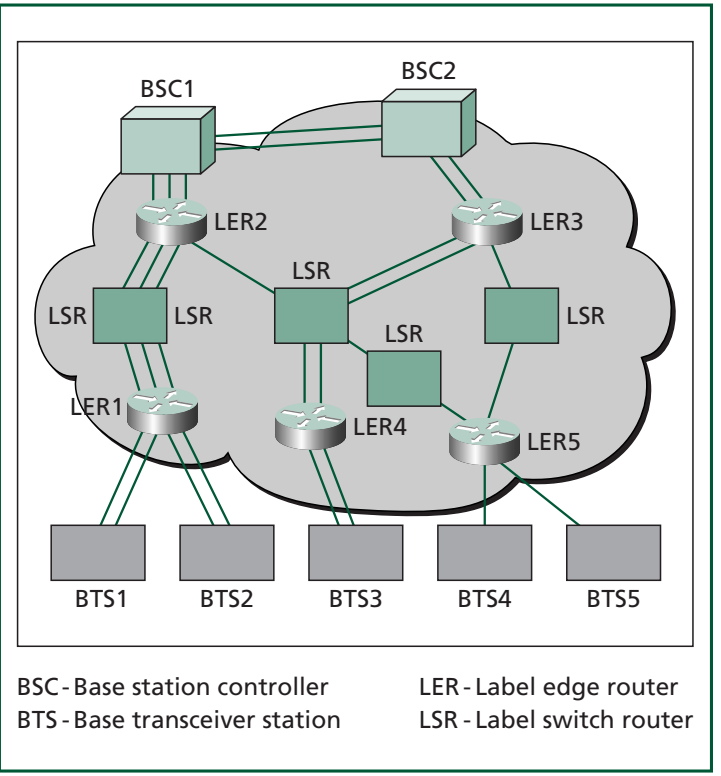

Figure 8.

MPLS architecture in a CDMA2000 RAN.

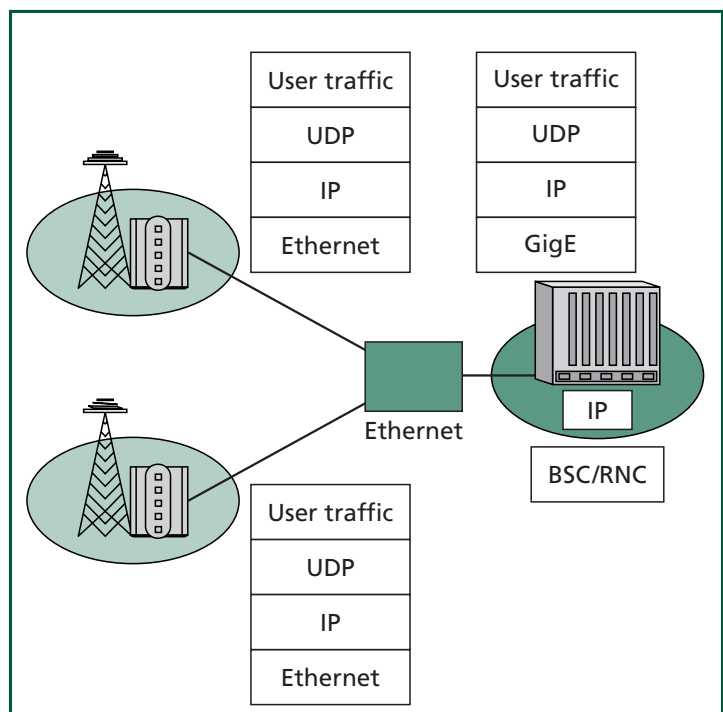

BSC - Base station controller RNC-Radio network controller IP - Internet protocol UDP-User datagram protocol

Figure 9.

Metropolitan area network using Ethernet.

need to care about the high overhead cost. An alternative way of carrying FP PDUs over Ethernet links is to use UDP/IP header compression and PPPMux. The resultant PPP frames are then carried over Ethernet. By employing such multiplexing, the overhead can easily be amortized among different voice streams, hence resulting in higher efficiency.

Note that an MPLS-based solution can also be used in the third topology. MPLSmux is a solution that can be used to multiplex several IP packets into a single MPLS frame. MPLSmux has the same overhead as with PPPMux and hence offers the same efficiency in multiplexing while providing superior QoS. The MPLSmux frame (essentially an MPLS frame) can then be carried over Ethernet on the LSP that is configured with the appropriate QoS requirements (e.g., voice, Web data, streaming). One can then map SI-Type_1 traffic onto LSP1, SI-Type_2 traffic into LSP2 and so forth using the L-LSP approach. Alternatively, one can also use the E-LSP approach where only one or two (for redundancy) LSPs are set up between one BTS and one BSC. The EXP bits within the MPLS header are used to provide different QoS treatments within 
the same LSP. We propose using the L-LSP approach since it is easier to manage. In order to guarantee the strict synchronized transmission over the air and account for a possible delay variation between frame inter-arrivals and among soft handoff legs, the dejittering buffer per traffic flow is required at the BTS, both for forward and reverse traffic. However, the BTS usually has limited resources for such buffering. The requirements for the QoS scheme must be such that the buffer will neither overflow nor starve. Hence the de-jittering buffer can absorb delay variations resulting from either unexpected or expected overhead traffic that is injected into the RAN. Such overhead traffic, for example, includes OA\&M traffic injected by the OA\&M elements and/or by the craft person and background audit traffic for system sanity checks.

\section{Summary of the End-to-End QoS Approach}

In summary, the QoS model for CDMA2000 packet data services consists of:

1. Parameters sent through the QOS BLOB from the MS. This covers air-link QoS and BTS-BSC QoS. Mobiles in assured mode can specify the following parameters:

a. Requested and acceptable user data rates-for the forward link and reverse link separately. The user data rate is the rate at which RLP is effectively being served.

b. Requested and acceptable data loss rate-for the forward link and reverse link separately. The data loss rate is the one measured after RLP error recovery procedures.

c. Requested and acceptable delay-for the forward link and reverse link separately. The delay is the total delay from the time when data enters RLP buffer to the time it is actually transmitted over the air.

In addition to the above QoS parameters, assured mode user can specify 13 levels of user priority within its subscription class. Users in the nonassured mode can also specify 13 priority levels. However, they cannot specify any QoS parameters.

2. DSCP marking on A10 and A11. While complying with DiffServ architecture is not a requirement (by the TSG-A standards group, at the time of writing), operators have the necessary framework needed for provisioning QoS using DiffServ. Again, MPLS can be used in conjunction with or as a replacement to DiffServ. It is possible that operators could simply over provision the A10/A11 interfaces instead of providing a QoS mechanism. The wireline part of the wireless bearer service classes may be implemented using DiffServ Architecture. However, the wireline network and the wireless network may be in different DiffServ domains. The PDSN/FA is the gateway between these two DiffServ domains. The PDSN/FA might need to translate the DSCPs and the PHBs between the two DiffServ domains, for proper treatments of packets across the domain boundary. The DSCPs might be the same between two domains, but PHBs could be different for the same DSCP in different DiffServ domains.

3. DSCP marking and MPLS tunnels on Abis/A3 interface. Based on the DSCP markings on the IP packet that encapsulates either $\mathrm{FCH}, \mathrm{SCHO}$, or SCH1 traffic, the corresponding LSP will be chosen. It is recommended that L-LSPs be used for each of the traffic types. As commented before, depending upon the traffic levels and bandwidth provisioned, DiffServ may turn out to be sufficient and MPLS may not be necessary. It is also possible that MPLS may not be used and some other layer 2 technology be used on the Abis/A3 interfaces. Then one needs to map the DSCP onto layer 2 QoS classes-e.g., 802.1p/q bits for an Ethernet-based solution, appropriate QoS classes for an ATM-based solution, MPLS class of service bits for an MPLS-based solution.

Using the QoS approach discussed in previous sections, QoS for a packet data call can be supported by CDMA2000 system. QoS for a VoIP type call can be handled in the current framework. However, two new service options are being defined specifically for VoIP, and the interaction between these service options and SO33 is subject to further study and is not covered in this paper.

\section{QoS in UMTS Networks}

Similar to CDMA2000, the UTRAN supports a number of different services (e.g., voice, data) and 
utilizes a number of physical channel types (dedicated, shared/common, control, paging) each with different bandwidth, loss, delay, and jitter requirements. It is desirable that a combination of planning and operational control (i.e., QoS) techniques be supported to allow the operator to enjoy some statistical multiplexing gain. Thus, the UTRAN transport network must support some form of differentiated services and admission control. These mechanisms must allow for the differentiation of traffic on a traffic-type basis (e.g., bearer frames, signaling messages). The bearer frames should be differentiated on a frame protocol basis (e.g., DCH FP, RACH FP, FACH FP). Differentiation by frame protocol types allows QoS parameters to match the unique characteristics for each unique physical channel type, as well as providing a framework for future frame protocol enhancements and additions. The transport network must support classification of these traffic flows and specific mechanisms to provide the desired characteristics (e.g., by mapping to a particular priority or using a particular per-hop queuing technique). The QoS approach described in earlier subsections, using differentiated services in MPLS, can equally be applied for UMTS networks. The primary and auxiliary service instance concept in CDMA2000 can be mapped to the primary and secondary PDP context concept in UMTS. In addition, the four service class categories (conversational, streaming, interactive, and background) are also defined in UMTS [1].

\section{Conclusion}

In this paper, we first described the current CDMA2000 RAN reference architecture, which is based on an ATM network. We then described how the protocol stacks will evolve when the IP-based RAN is introduced. Next, we gave an overview of the QoS framework in DiffServ-enabled and MPLSenabled IP networks and how they can be combined. Later, we described the QoS approach for an IP-based RAN. We provided a summary of the existing support for QoS on the backhaul and over the air in the current 3 GPP2 standards. We proposed using MPLS in conjunction with DiffServ over the A8/A10 interfaces. On the Abis/A3 interface, we proposed classifying traffic in RAN into five categories and suggested appropriate DSCP markings for such traffic. We suggested using different LSPs to carry traffic marked with different DSCP in order to meet their QoS requirements. We then investigated three sample IP-based RAN topologies and discussed how DiffServ and MPLS can be used together to provide the desirable QoS features. Finally, we briefly commented on how the QoS framework described for CDMA2000 can be applied to UMTS system also.

\section{*Trademark}

CDMA2000 is a trademark of the Telecommunications Industry Association.

\section{References}

[1] 3rd Generation Partnership Project, Technical Specifications for Services \& System Aspects: QoS Concept and Architecture, 3GPP TS23.107, June 2002, <ftp://ftp.3gpp.org/specs/latest/Rel-5/ 23_series/23107-550.zip>.

[2] 3rd Generation Partnership Project 2, "Interoperability Specification (IOS) for CDMA2000 Access Network Interfaces," IS-2001, June 2001, <http://www.3gpp2.org/Public_html/specs/ A.S0001-A_v2.0.pdf>.

[3] 3rd Generation Partnership Project 2, "Wireless IP Network Standard," IS-835 (3GPP2 P.S0001-B), Jan. 2002, <http://www.3gpp2.org >.

[4] L. Andersson, P. Doolan, N. Feldman, A. Fredette, and B. Thomas, "LDP Specification," RFC-3036, IETF, Jan. 2001, $<$ http://www.ietf.org/rfc/rfc3036.txt>.

[5] J. Ash, Y. Lee, P. Ashwood-Smith, B. Jamoussi, D. Fedyk, D. Skalecki, and L. Li, "LSP Modification Using CR-LDP," RFC-3214, IETF, Jan. 2002, $<$ http://www.ietf.org/rfc/rfc3214.txt>.

[6] D. Awduche, L. Berger, D. Gan, T. Li, V. Srinivasan, and G. Swallow, "RSVP-TE: Extensions to RSVP for LSP Tunnels," RFC-3209, IETF, Dec. 2001, <http://www.ietf.org/rfc/rfc3209.txt>.

[7] F. Baker (ed.), "Requirement for IP Version 4 Routers," RFC-1812, IETF, June 1995, $<$ http://www.ietf.org/rfc/rfc1812.txt>.

[8] P. Bender, P. Black, M. Grob, R. Padovani, N. Sindhushayana, and A. Viterbi, 
“CDMA/HDR: A Bandwidth Efficient High Speed Wireless Data Service for Nomadic Users," IEEE Commun. Mag., 38:7 (2000), 70-77.

[9] S. Blake, D. Black, M. Carlson, E. Davies, Z. Wang, and W. Weiss, "An Architecture for Differentiated Services," RFC-2475, Dec. 1998, $<$ http://www.ietf.org/rfc/rfc2474.txt>.

[10] R. Braden (ed.), "Resource ReSerVation Protocol (RSVP)-Version 1 Functional Specification," RFC-2205, IETF, Sept. 1997, <http://www.ietf.org/rfc/rfc2205.txt>.

[11] R. Braden, D. Clark, and S. Shenker, "Integrated Services in the Internet Architecture: an Overview," RFC-1633, IETF, June 1994, <http://www.ietf.org/rfc/rfc1633.txt>

[12] S. Casner and V. Jacobson, "Compressing IP/UDP/RTP Headers for Low-Speed Serial Links," RFC-2508, IETF, Feb 1999, <http://www.ietf.org/rfc/rfc2508.txt>.

[13] S. Deering and R. Hinden, "Internet Protocol, Version 6 (Ipv6) Specification," RFC-2460, IETF, Dec. 1998, <http://www.ietf.org/rfc/rfc2460.txt>.

[14] M. Degermark, B. Nordgren, and S. Pink, "IP Header Compression," RFC-2507, IETF, Feb. 1999, $<$ http://www.ietf.org/rfc/rfc2507.txt>.

[15] S. Dixit, Y. Guo, and Z. Antoniou, "Resource Management and Quality of Service in Third-Generation Wireless Networks," IEEE Commun. Mag., 39:2 (2001), 125-133.

[16] J. Heinanen, F. Baker, W. Weiss, and J. Wroclawski, “Assured Forwarding PHB Group," RFC-2597, IETF, June 1999 <http://www.ietf.org/rfc/rfc2597.txt>.

[17] V. Jacobson, K. Nichols, and K. Poduri, "An Expedited Forwarding PHB," RFC-2598, IETF, June 1999, <http://www.ietf.org/rfc/rfc2598.txt>.

[18] F. Le Faucher (ed.), L. Wu, B. Davie, S. Davari, P. Vaananen, P. Krishnan, P. Cheval, and J. Heinanen, "MPLS Support of Differentiated Services," RFC-3270, IETF, May 2002, <http://www.ietf.org/rfc/rfc3270.txt>

[19] K. Nichols, S. Blake, F. Baker, and D. Black, "Definition of the Differentiated Services Field (DS Field) in the IPv4 and IPv6 Header," RFC-2474, IETF, Dec. 1998, <http://www.ietf.org/rfc/rfc2474.txt>.
[20] R. Pazhyannur, I. Ali, and C. Fox, "PPP Multiplexing," RFC-3153, IETF, Aug. 2001, $<$ http://www.ietf.org/rfc/rfc3153.txt>.

[21] J. Postel (ed.), "Internet Protocol," STD5, RFC-791, IETF, Sept. 1981, $<$ http://www.ietf.org/rfc/rfc791.txt>.

[22] E. Rosen, A. Viswanathan, and R. Callon, "Multiprotocol Label Switching Architecture," RFC-3031, IETF, Jan. 2001, <http://www.ietf.org/rfc/rfc3031.txt>.

[23] Telecommunications Industry Association, "CDMA2000 High Rate Packet Data Air Interface Specification," TIA/EIA IS-856, Dec. 2001, <http://www.3gpp2.org/Public_html/specs/ C.S0024-0_v3.0.pdf>.

[24] Telecommunications Industry Association, "Data Service Options for Spread Spectrum Systems-Addendum 2: CDMA2000 HighSpeed Packet Data Service Option," TIA/EIA IS-707-A-2.12, Aug. 2000, <http://www.3gpp2.org/Public_html/specs/ C.S0017-0-2_v2.0.pdf>.

[25] Telecommunications Industry Association, "Introduction to CDMA2000 Standard for Spread Spectrum Systems, Release C," TIA/EIA IS-2000, May 2002, <http://www.3gpp2.org/Public_html/specs/ C.S0001-C_vl.0.pdf>.

(Manuscript approved July 2002)

MOOI CHOO CHUAH is a technical manager in the

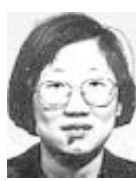
Advanced Technologies Division of Bell Labs at Holmdel, New Jersey. She holds a Ph.D. in communication systems and theory from the University of California, San Diego. Dr. Chuah has over 30 publications covering topics in media access control, power control algorithms, mobility management, and TCP performance. She has been awarded 10 patents and has 45 additional patents pending.

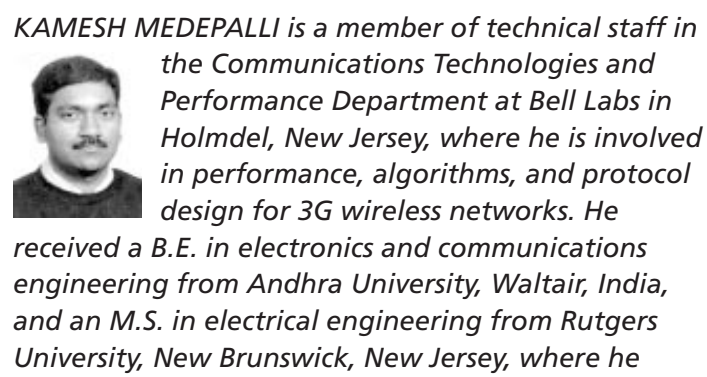


worked as a graduate research assistant at WINLAB.

His research interests include signal processing,

communication theory, and communication networks.

SE-YONG PARK is a member of technical staff in the

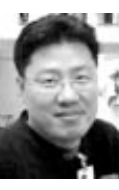

AMPS/PCS Systems Engineering

Department, Wireless Networks Group, at

Lucent Technologies in Whippany, New

Jersey. As a systems engineer, he has been

involved in RAN architecture, including

frame relay, ATM interface between base stations and the MSC, and IP RAN architecture for $3 G$ wireless networks. He received a B.E. from SungKyunKwan University in Seoul, Korea, and an M.S.E. in electrical engineering and computer science from the University of Michigan at Ann Arbor. He is currently in the Ph.D. program in computer engineering at the State University of New York at Stony Brook. His research interests include wireless RAN architecture, transport protocols, and low-power mobile system design.

JIANSONG WANG is a member of technical staff in the

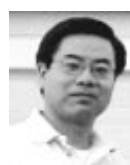
Wireless System Architecture and $3 G$ IP Platform Architecture Department at Lucent Technologies in Indian Hill, Illinois. He holds an M.S. in telecommunication engineering from Columbia University in New York City. He has worked with wireless system performance, Flexent ${ }^{\circledR}$ wireless router platform architecture, and UMTS RNC architecture. He is currently working on Flexent packet core network architecture and IX RNC architecture. 